\title{
AC 2012-5482: ON THE IMPLEMENTATION OF OPEN SOURCE CFD SYSTEM TO FLOW VISUALIZATION IN FLUID MECHANICS
}

\author{
Ricardo Medina, California State University, Los Angeles \\ Mr. Ashkan Motamedi, California State University, Los Angeles \\ Dr. Murat Okcay, Interactive Flow Studies Corporation
}

Murat Okcay, CEO, obtained his doctorate in mechanical engineering, specializing in fluid mechanics, in 1993 from Bristol University, England. After several years as a lecturer teaching fluid mechanics in the classroom and laboratories at the University he joined Smiths Industries Plc. and has continually pushed the envelope in the field of fluid mechanics as a Senior Mechanical Design Engineer, publishing papers and receiving patents for his designs. He was then recruited by Allied Signal, in the U.S., as a Project Engineer during which time he completed his master's of business administration (MBA) at the University of Arizona in Tucson. He then moved to Minneapolis working as a Sales Manager at TSI responsible for sales and marketing of research-grade PIV products in the U.S., Canada and Brazil. As the PI, he directed an NSF SBIR-funded project with Phase I and Phase II grants. During Phase II, the company generated more than $\$ 0.25$ million income from sales and received Phase $2 \mathrm{~B}$ funding. He published several papers in this field. He not only has strong business background and technical expertise in mechanical design and teaching fluid mechanics, but he also provides his vision, strategic direction, very broad experience, nationally and internationally, and industry contacts to Interactive Flow Studies.

\section{Dr. B. Uygar Oztekin, Interactive Flow Studies Corporation \\ Dr. Gustavo Borel Menezes, California State University, Los Angeles}

Gustavo Borel Menezes is an Assistant Professor of civil engineering at California State University, Los Angeles, where he has been since 2009. During 2007-2009, he was a Postdoctoral Research Fellow at Spelman College. He received a B.S. from Universidade Federal de Minas Gerais in 2001, and an M.S. from the University of North Carolina, Charlotte, in 2004. He received his Ph.D. in infrastructure and environmental systems in 2007 from UNC Charlotte. His research interests are in the area of geoenvironmental engineering, focusing on fate and transport of contaminant in the subsurface. His recent work looks into characterization of transport under unsaturated and low conductivity condition using steadystate centrifugation method. He is an Associate Editor of the ASCE Journal of Energy Engineering and a board member of the International Society for Environmental Geotechnology (ISEG).

\section{Dr. Arturo J. Pacheco-Vega, California State University, Los Angeles}

Arturo Pacheco-Vega completed his undergraduate studies in mechanical engineering at the University Iberoamericana in Leon, Mexico. After receiving master's degrees in mechanical engineering at the University of Guanajuato in Salamanca, Mexico, and at the University of Notre Dame, he received his Ph.D. in mechanical engineering from the University of Notre Dame in early 2002, funded by a FulbrightCONACYT-Garcia Robles scholarship. From 2003 to 2008, he was a tenured full-time Associate Professor at the Department of Chemical Engineering of the Autonomous University of San Luis Potosi (UASLP) in San Luis Potosi, Mexico. Currently, Pacheco-Vega is a tenured faculty member in the Department of Mechanical Engineering at California State University, Los Angeles. His research is situated broadly in the fluid mechanics and heat transfer fields. His current interest is in simulation, optimization and control of thermal systems, and the applications of soft computing techniques in complex systems. 


\title{
On the Implementation of Open Source CFD System to
}

\author{
Flow Visualization in Fluid Mechanics
}

\begin{abstract}
In this contribution, we combine computational fluid dynamics (CFD) tools with particle image velocimetry (PIV) experiments, to supplement the traditional teaching method. The objective is to provide a well-rounded experience for engineering students to learn and apply fluid mechanics concepts in the design of physical systems. In this approach, the integration of theory, experimental analysis, and mathematical/numerical models of engineering systems is done through an open-source CFD software named OpenFOAM. This numerical software, which is grounded on the finite-volume technique, is to be linked to an educational interactive flow visualization system (i.e., FlowCOACH), currently used to provide students with opportunities for visual appreciation of the flow phenomena. A main advantage of using OpenFOAM is that building the geometry and setting the boundary and initial conditions for the model is straightforward. The problem of flow around a squared obstruction, inside a channel, is used to assess the accuracy of the numerical solution obtained from OpenFOAM. Comparison of the results against a well-known commercial CFD software indicate that OpenFOAM is a reliable numerical tool that can be used to teach students the topic of fluid mechanics.
\end{abstract}

\section{Introduction}

The study of fluid mechanics is essential to many applications, ranging from electronic cooling to power generation systems, where reliable engineering equipment needs to be adequately designed and built. It is for this reason that engineering students must have a good understanding of the concepts behind the design and use of fluid-flow systems. Although theory is absolutely necessary to understand the underlying mechanisms of fluid flow, and experiments form an excellent supplement to provide students with opportunities for visual appreciation of the complexity of flow phenomena, the ever increasing challenge of improving the design of engineering systems, demonstrates the great importance to integrate computer simulations in the classroom. Integration of computer models to many application areas in fluid mechanics has proven to be successful ${ }^{[1,2,3]}$. While experimental data are useful for specific scenarios, engineering design of fluid flow systems often require large sets of simulations in which a particular design is tested under different conditions of operation. A cost-effective solution is the use of computational fluid dynamics (CFD) simulations.

CFD is a fast growing branch of fluid mechanics, in which one is able to model physical systems by numerical integration of the governing equations. It supplements theoretical analysis of fluid mechanics by allowing the professionals to obtain numerical solutions to complex fluidflow phenomena such as turbulent, transonic, two-phase, and reactive flow, which would otherwise be difficult to solve analytically or experimentally. 
The traditional approach to the teaching of fluid mechanics involves instilling knowledge of theoretical concepts in the classroom that is supplemented by laboratory experiments; this offers the student the opportunity to learn the basics of fluid flow, and apply them in a laboratory setting. Through the use of CFD the students can perform in-depth analysis of engineering problems, thus attaining a better understanding of the theory. We are currently in the process of implementing a new approach to the teaching of fluid mechanics that combines theory, physical experiments and CFD analysis. This new approach offers students a well-rounded fluid mechanics education, which relies on the coupling of FlowCoach (a portable computercontrolled flow visualization equipment developed by Interactive Flow Studies LLC), with the open-source OpenFOAM CFD software. First, the manuscript presents general information on the OpenFOAM software. Then, description of a specific fluid mechanics problem, that can be analyzed experimentally, is presented and numerically solved using OpenFOAM. Validation of OpenFOAM results is carried out by comparison to the solutions obtained by the well-known commercial software COMSOL Multiphysics, and to experimental visualization data obtained from the FlowCOACH system, which will be described in a later section. The study ends with some concluding remarks.

\section{Open Source CFD Software}

Open Field Operation and Manipulation (OpenFOAM) is a free, open source CFD software package produced by OpenCFD Ltd (www.openfoam.com) under the GNU general public license (GPL). It is an object-oriented software designed for the analysis of a large range of engineering topics including complex fluid flows, chemical reactions, turbulence, heat transfer, solid dynamics, and electromagnetics. The software package is grounded on the method of finite volumes, which allows discretizing the governing continuum equations into a set of algebraic equations than can be solved on the computer via matrix-vector calculations. In the finite volume method, the values of the corresponding variables are computed on specific points (i.e., nodes) of small control volumes that comprise the discretized computational domain. The integral form of the governing equations is then evaluated on each elemental volume to generate the solution set for the problem. A main advantage of the finite volume technique is that it can be easily formulated on unstructured meshes. Additional details on the method of finite volumes, and CFD analysis, can be found in the books of Tannehill et al. ${ }^{[4]}$, and Malalasekera and Verseeg ${ }^{[5]}$.

The OpenFOAM software comes with subroutines denominated 'solvers' that can be applied to the solution of incompressible flows, multiphase flows, combustion, buoyancy-driven flows, conjugate heat transfer, and compressible flows, among other fields. These predefined solvers can be easily implemented to suit specific problems. By being 'open,' OpenFOAM provides users with the complete freedom to customize, adjust, and extend its default functionality, so that, experienced users can also develop new solvers. The software is designed in a modular way by which collections of functionalities are compiled into their own library and executable applications can then be linked to these libraries upon run-time. Additionally, the software has a large number of pre-defined solver applications, utilities libraries, meshing tools, and pre-and post-processing tools, which the user can implement to simulate a number of specific engineering problems. One of the main features of its toolbox is parallel processing, which can become useful when studying large and complex-geometry models. Another important feature is 
the number of numerical schemes from which the user can choose for the calculations of the specific case, which allows the user to study the effects of different discretization techniques and different numerical schemes on the same problem and thus optimize the system.

Disadvantages of the application of OpenFOAM include the large CPU-time required for some cases and the lack of official documentation describing the implementation of the software; particularly, an in-depth description, including definitions of some of the parameters that are used in the pre-defined solvers. Because there is no such documentation readily available, one must usually rely on the help offered by community-based web forums, making it difficult to learn to a level which would allow the user to make full use of all the capabilities offered by the software.

\section{Problem Description}

\section{Mathematical model}

The problem considered here to illustrate the capability of OpenFOAM is the flow of water inside a narrow rectangular channel and around an obstruction shaped as a square. A physical prototype of this problem, of which a two-dimensional sketch of its geometry is shown in Fig. 1, has been recently used in the FlowCOACH experimental setup as a 'model insert' to provide students at CSULA supplemental information through visual appreciation of the flow in a laboratory setting ${ }^{[6]}$. In reference to Fig. 1, the channel geometry is comprised of three different sections: (1) a diverging section with dimensions $27 \mathrm{~mm}$ and $80 \mathrm{~mm}$ at the end points, and a length of $90 \mathrm{~mm}$ in the streamwise-direction ( $x$-direction), (2) a squared section, which corresponds to the model insert in the FlowCOACH device, with dimensions of $80 \mathrm{~mm} \times 80$ $\mathrm{mm}$, and (3) a converging section with dimensions: $80 \mathrm{~mm}$ and $42 \mathrm{~mm}$ at the end points and a length of $27 \mathrm{~mm}$. The squared-obstruction of the model insert has dimensions of $20 \mathrm{~mm} \times 20$ $\mathrm{mm}$ and is located at $x=99 \mathrm{~mm}$ from the origin of the coordinate system. The channel depth is constant and equal to $5 \mathrm{~mm}$ in the $z$-direction.

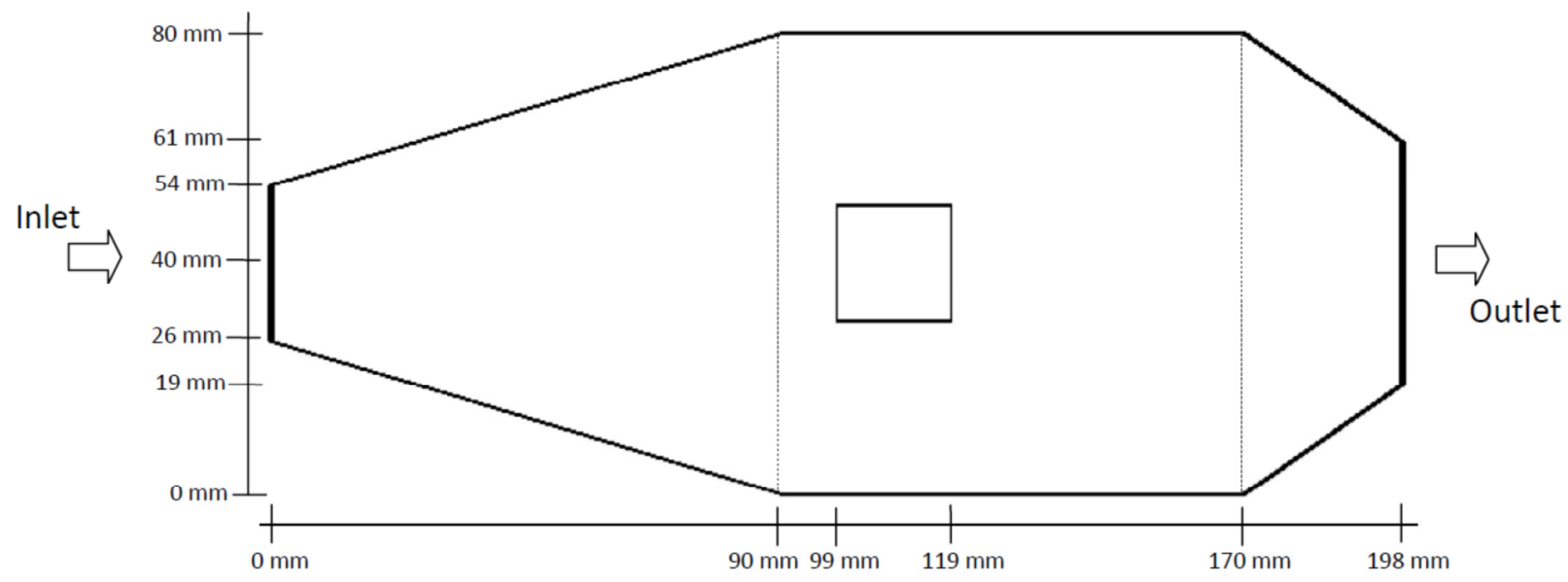

Figure 1. Two-dimensional view of a schematic of the system under analysis. 
The mathematical model is built upon the underlying principles of conservation of mass and Newton's laws of motion (for a detailed derivation of these equations see Malalasekera et al. ${ }^{[5]}$, Pnueli et al. ${ }^{[7]}$, and Ferziger et al ${ }^{[8]}$ ). Neglecting body forces, the continuity and momentum equations for an incompressible, laminar flow of a Newtonian fluid with constant properties, under steady-state conditions, can be written in vector form as

$$
\begin{aligned}
\nabla \cdot \mathbf{u} & =0, \\
(\mathbf{u} \cdot \nabla) \mathbf{u} & =-\frac{1}{\rho} \nabla p+\nu \nabla^{2} \mathbf{u} .
\end{aligned}
$$

In Eqs. (1) and (2), commonly known as continuity and Navier-Stokes (N-S) equations, $\mathbf{u}=$ $(u, v, w)$ is the three-dimensional Cartesian velocity vector of components $u, v$, and $w$, in the directions $x, y$, and $z$, respectively; $p$ is the pressure, $\rho$ is the fluid density, and $v$ is the kinematic viscosity. The solution of these equations is complex and difficult because (a) the momentum equation has a non-linear term, namely the convection term, and (b) there exists a coupling of velocity and pressure in a single equation ${ }^{[5,9,10]}$. The boundary conditions for the fluid flow problem at hand are the typical no-slip and impermeable conditions at all the walls, an influx velocity condition $u_{i n}$ at the inlet, and a zero-pressure condition at the outlet.

\section{Numerical schemes}

Although the section concentrates on the application of OpenFOAM, a mirror procedure was carried out for the assessment via the commercial software COMSOL Multiphysics (www.comsol.com), which is described later in this section. To save computation time, only half of the domain is simulated, imposing a symmetry-plane at $z=2.5 \mathrm{~mm}$ location. The equations are discretized on the domain by using the finite volume method (FVM). Although other discretization schemes for partial differential equations are given in Malalasekera et al. ${ }^{[5]}$, Ferziger et al. ${ }^{[8]}$, and Rusche ${ }^{[13]}$, the FVM is favorable in fluid-flow problems because it offers a clear physical interpretation of the discretized equations. Here the domain is discretized with hexahedral elements of finite volume in a structured mesh. A typical mesh used for simulations in OpenFOAM is presented in Fig. 2, along with that used for COMSOL. A fine mesh is used to ensure that the fluid-solid interactions are captured and resolved. The mesh is constructed as follows: the divergent section has 180 elements in the $x$-direction and 160 elements in the $y$ direction. The mid-section has 160 elements in both the $x$ - and $y$-directions. The convergent section has 50 elements in the $x$-direction and 160 elements in the $y$-direction. The squaredobstruction is modeled as an empty space with wall-like properties at the edges. The total number of mesh points for the domain is 182,400 . As noted earlier, the momentum equation has the convection term which is non-linear. A substantial amount of research ${ }^{[5,9,12,14]}$ has been done to find an optimized discretization scheme for the non-linear term of the momentum equation. However, because of its simplicity and accuracy, the upwind scheme has been widely implemented in CFD calculations ${ }^{[5]}$. For the solution in OpenFOAM a Gauss Upwind scheme is chosen for the treatment of the non-linear term; the remaining terms use a Gauss linear scheme.

In OpenFOAM, the incompressible Navier Stokes equations are solved by implementing the SIMPLE (Semi-Implicit Method for Pressure Linked Equations) algorithm, developed by Spalding and Patankar in $1972^{[5,8-11]}$. The SIMPLE algorithm solves the governing equations iteratively by guessing an initial value for the pressure field, from which an intermediate-value of 


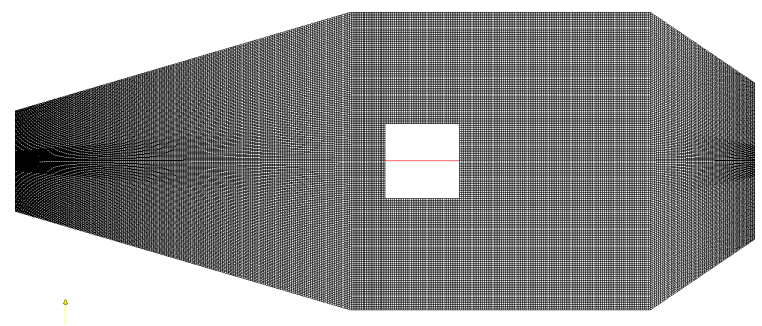

(a)

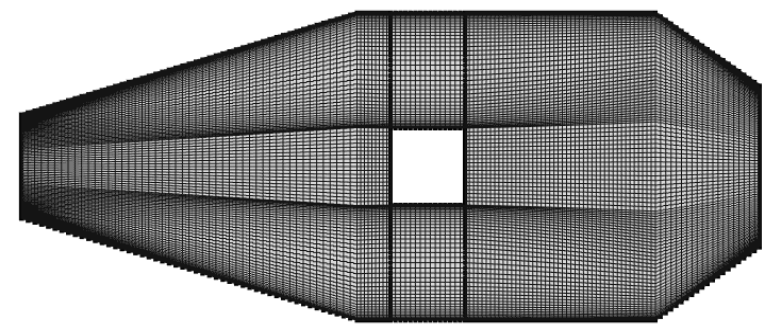

(b)

Figure 2. Typical meshing of the domain. (a) OpenFOAM. (b) COMSOL Multiphysics.

the velocity field is obtained from solution of the momentum equations. Velocity- and pressurecorrection terms are then calculated from the approximated velocity values given that the new velocity values must satisfy the continuity equation. This process is repeated until the solution has reached an acceptable predefined level of error (or tolerance). Detailed description of this method can be found in Malalasekera et al. ${ }^{[5]}$, Ferziger et al. ${ }^{[8]}$, Patankar ${ }^{[10]}$ and Galpin et al. ${ }^{[11]}$.

For the assessment of the results via COMSOL Multiphysics, the governing equations were discretized on the domain and solved by the finite element method. In this technique, the computational domain is divided into a set of discrete subdomains, denominated elements, each with specific shape and mathematical properties; i.e., the approximation functions (and corresponding coefficients) of a series expansion that describes the solution of the governing equations. A weighted-integral process of the residual between the approximated and the original equations over each element generates a set of algebraic relations among the unknown parameters of the approximation, and hence, the solution by a matrix-vector operation. The geometry is constructed using a three-dimensional mesh with quadrilateral elements along the boundary and hexahedral elements inside the domain. To ensure accurate results while maintaining a manageable CPU time, more dense meshing is using near all the wall boundaries. For the construction of the mesh in COMSOL, 160 elements were used in the $y$-direction throughout the domain, with exception of the region where the squared-obstruction is modeled, where only 120 elements were used. A number of 173 elements were used the $x$-direction, giving a total number of elements of 78240. The steady-state problem was solved by COMSOL with the iterative scheme known as Generalized Minimum Residual Method (GMRES), for which the relative tolerance was set to $10^{-6}$. For both OpenFOAM and COMSOL several grids were tested in order to ensure grid independence of the corresponding results.

\section{Results and Analysis}

The numerical results obtained from OpenFOAM are shown qualitatively in the form of velocity fields in Fig. 3, and streamlines in Fig. 4, at the symmetry plane. On the other hand, the same results are shown quantitatively, in terms of the streamwise velocity ( $x$-direction), in Fig. 5. For purposes of direct comparison, in the figures the OpenFOAM results are shown side-by-side with the numerical solutions computed by COMSOL. From Figs. 3-5, it is clearly seen that, as expected, the results obtained from OpenFOAM are very close to those of COMSOL, and 
describe the fluid flow of water in the channel showing similar patterns for the velocity field and the streamlines. Both numerical solutions present regions of recirculation due to the large slope of the divergent section which produces an adverse pressure gradient, causing the flow to separate and reverse its direction. Due to these recirculation areas at the development-flow region of the channel, the mean velocity in the central region first accelerates until later decelerating due to the presence of the squared-obstruction. Also expected are the flow recirculation regions at the top and bottom edges of the object and at the region behind it. It is to be noted that these recirculation effects are consistent with those obtained by the visualization experiment presented in Fig. 7. It should also be noted that, although not shown here, the recirculation effects obtained numerically in the diverging region of the channel, are absent in the actual physical system. This is due to the fact that the experimental prototype contains a set of veins that re-direct the flow to avoid flow separation in this region. This effect has an impact on the flow downstream; thus, smaller recirculation regions would be expected as a consequence. Although close, there are some differences between the results from the numerical schemes. The results from COMSOL show a larger area of flow separation in the divergent inlet channel; OpenFOAM also shows that effect, but to a lesser extent.

Figure 5 shows, quantitatively, that the numerical results from OpenFOAM and COMSOL follow the same pattern and are very close. For purposes of comparison, plots of the $x$ component of velocity at different cross-sections; i.e., at (a) $90 \mathrm{~mm}$, which is the starting point of the model insert; at (b) $99 \mathrm{~mm}$, at the left boundary of the squared-obstruction; at (c) $130 \mathrm{~mm}$,

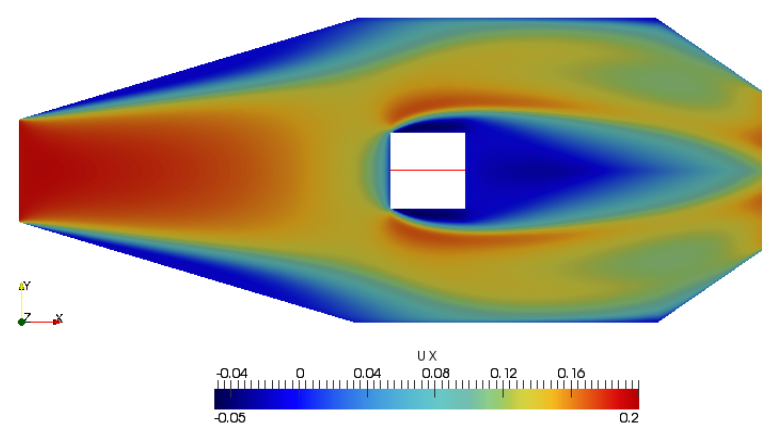

(a)

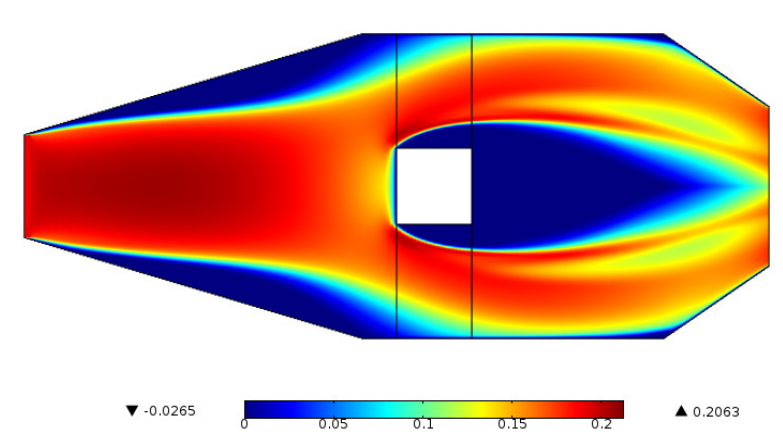

(b)

Figure 3. Velocity field, x-component: (a) OpenFOAM (b)COMSOL Multiphysics.

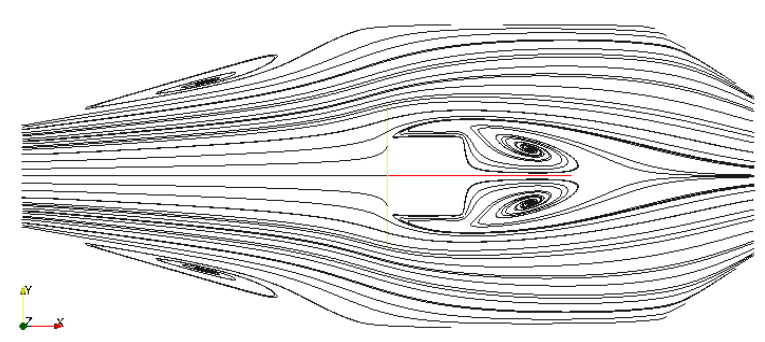

(a)

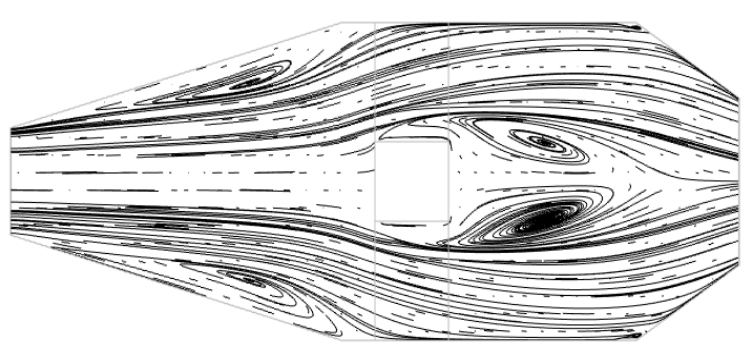

(b)

Figure 4. Streamlines at the symmetry plane $(z=2.5 \mathrm{~mm})$. (a) OpenFOAM, (b) COMSOL. 


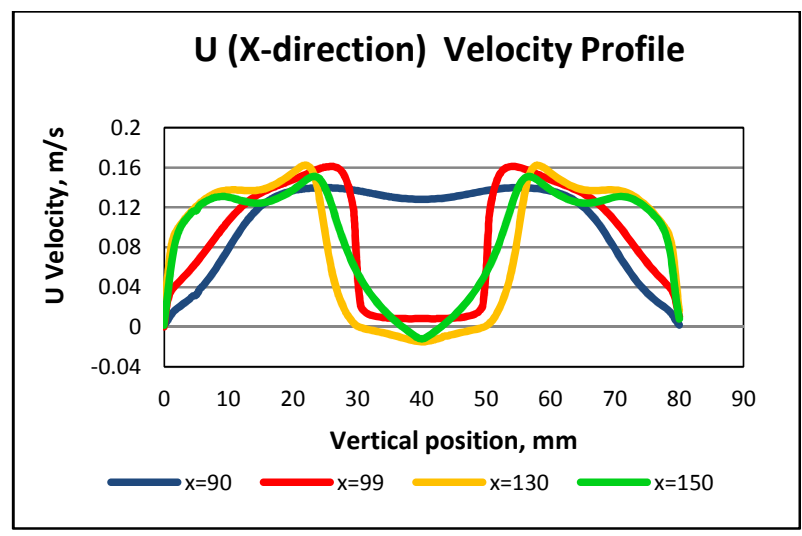

(a)

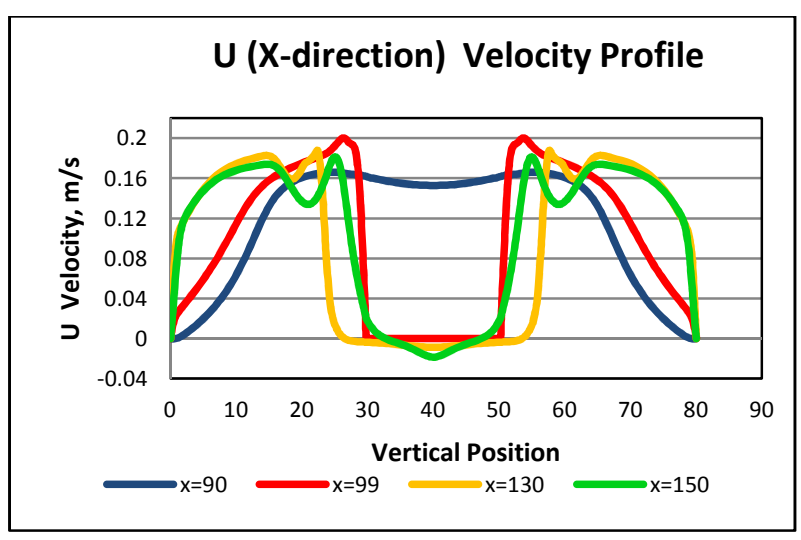

(b)

Figure 5. Streamwise-component of the velocity at different cross-sections along the channel. (a) OpenFOAM. (b) COMSOL.

which correspond to the plane located at midpoint of the model insert; and at (d) $150 \mathrm{~mm}$, at the recirculation zone are illustrated in this figure. From the figure it is observed that, in general, the velocities in the $x$-direction obtained from COMSOL are slightly higher than those results obtained by OpenFOAM. There are several possible explanations for the difference in the results which include the difference in the discretization schemes and number of meshing points and the type of interpolation methods used to obtain the velocity fields in the domain.

\section{Experimental System and Analysis of Flow Visualization Results}

FlowCoach ${ }^{\mathrm{TM}}$ is an educational flow visualization and analysis system designed and produced by Interactive Flow Studies Corp. to be used in a classroom or laboratory. The Flowcoach visualization instrument, shown in Fig. 6, can be used with a variety of inserts containing different obstruction geometries, allowing for the study of different flow phenomena in different geometries. Neutrally-buoyant polyamide seeds (particles) are added to the water to enhance flow visualization. The seeds reflect light emitted by attached LED lights, giving the students an excellent opportunity to visually examine the effects the obstruction has on the flow. For the problem being analyzed, the flow rate passing through the channel is set at 0.4 gpm which gives a uniform inlet velocity of $0.1869 \mathrm{~m} / \mathrm{s}$ (in the $x$-direction).

Flowcoach uses particle image velocimetry (PIV), a technique used to obtain instantaneous velocities of the flow at given points in space. A mounted camera captures a short video of the flow around the square obstruction (other geometries are available) that is decomposed into individual frames. The PIV technique compares sequential frames to approximate the distance a group of particles travels over an area. Using this approximated distance and the time between each frame (determined from the video capture speed), the instantaneous velocity is determined at a point. This technique is repeated to analyze the entire region and the result is the instantaneous velocity vector field. Though Flowcoach provides students with opportunities for visual appreciation and an excellent qualitative analysis of the flow, several factors that might contribute to poor results include: the camera settings such as positioning and capture speed, 


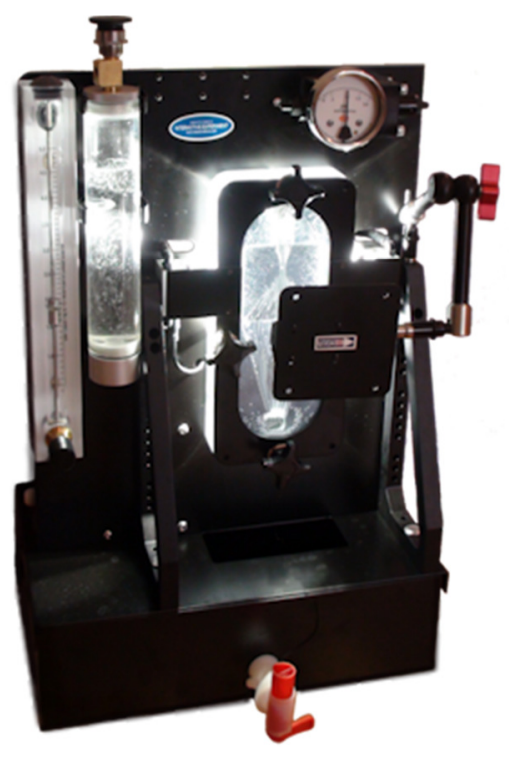

Figure 6. FlowCOACH by Interactive Flow Studies Corporation.

lighting, quantity of seeds, overall quality of video captured, correct adjustment of inputs and the efficiency of the integrated PIV software.

Experimental tests of the flow around the aforementioned squared-obstruction were carried out using FlowCOACH. The value of the inlet velocity for the experiment corresponds to that considered for the numerical simulations $u_{\text {in }}=0.1967 \mathrm{~m} / \mathrm{s}$. Figure $7 \mathrm{a}$ shows the flow field in terms of the streamlines around the squared-obstruction. From this image the regions where the fluid is stagnant at the leading edge of the object, the development of the boundary layer and consequent flow separation at the top and bottom surfaces, and the recirculation bubble at the trailing edge of the obstruction can be clearly seen. A qualitative comparison of the experimental observations to the OpenFOAM numerical simulations, on the other hand, is shown in Fig. $7 \mathrm{~b}$. The figure reveals that the solution obtained via CFD depicts, fairly accurate, the true physical characteristics of the flow in the region around the object. By looking closer, one can perceive some slight differences between the superimposed numerical solution and the reflected neutrallybuoyant particles. These differences are thought to be due to the fact that the numerical model has been constructed without taking into account the directional veins that the physical prototype; named, the model insert, contains to eliminate the recirculation zones at the divergent region of the channel, which are shown in the numerical solutions of Figs. 3 and 5.

\section{Concluding Remarks}

Applications of fluid mechanics are found in many areas of civil and mechanical engineering. Thus, an in-depth understanding of FM is a pre-requisite to engineers working in these fields. This paper proposes a new approach to teaching fluid mechanics concepts that integrates computation fluid dynamics (CFD) simulations to the conventional theory/laboratory method. While analytical solutions that are taught in theory-focused lessons can be applied to elementary problems, understanding and design of more advanced systems cannot be achieved through these 


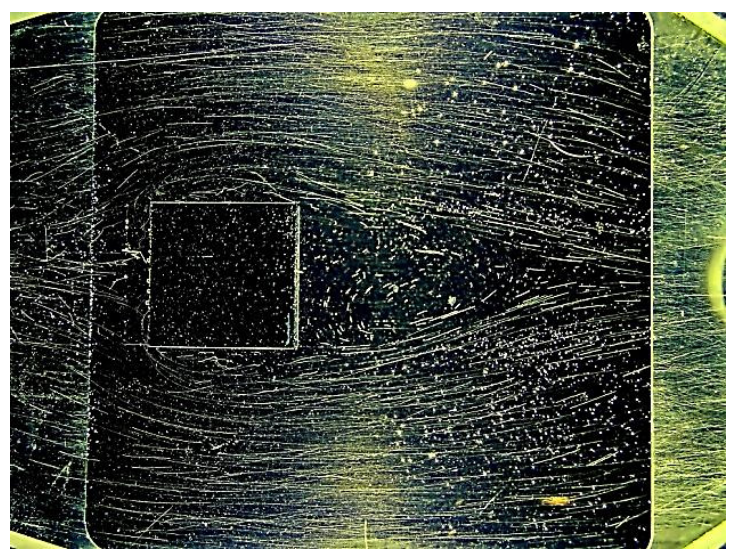

(a)

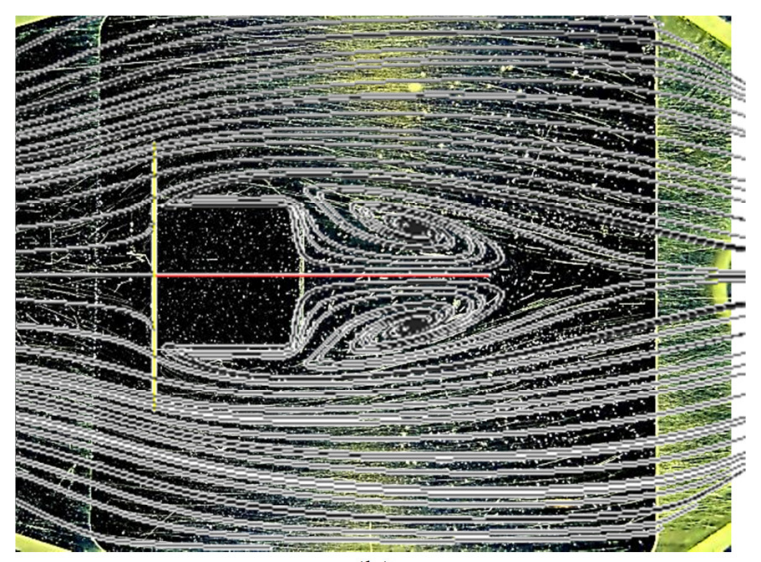

(b)

Figure 7. Flow visualization of the flow field. (a) Original image. (b) OpenFOAM superimposed streamlines.

solutions alone. Testing and measurements of experimental settings are often an effective alternative. In most cases, however, accurate measurements of fluid pressure and velocity fields are either very expensive or impossible. In these cases, one can only rely on validated numerical solutions, such as CFD simulations. Through this integrated approach, students learn the benefitsof each method and how they can be applied in the design of complex fluid-flow systems. An open-source software known as OpenFOAM was the CFD software of choice. In addition to being free, the large number of pre-defined solvers included and the capability for user-defined solvers make OpenFoam suitable for educational and research purposes. Comparison of OpenFOAM with COMSOL, a widely-used commercial software, has proven OpenFOAM to be very accurate, reliable and easy to implement. Experimental analysis was performed using a flow visualization device named FlowCoach. A qualitative comparison of FlowCoach and OpenFoam results indicates that the CFD results are fairly accurate. The proposed integrated approach is currently being implemented at California State University Los Angeles. It is expected that it will significantly enhance student learning of abstract and complex concepts of fluid mechanics.

\section{Acknowledgements}

This material is based upon work supported by the National Science Foundation NSF-SBIR phase II grant (IIP-0844891), and partially supported by NSF-funded CREST center (HRD0932421). Any opinions, findings, and conclusions or recommendations expressed in this material are those of the author(s) and do not necessarily reflect the views of the National Science Foundation. 


\section{References}

[1] Friedrich, J. "Object-oriented design and implementation of CFDLab: a computer assisted learning tool for fluid dynamics using dual reciprocity boundary element methodology." Computers and Geosciences, Volume 25, 1999, pp. 785-800.

[2] Stern, F., Xing, T., Yarbrough, D.B., Rothmayer, A., Rajagopalan, G., Otta, S.P., Bhaskaran, R., Smith, S., Hutchings, B., Moeykens, S. "Hands-on CFD educational interface for engineering courses and laboratories." Journal of Engineering Education. Volume 95, No. 1, 2006, pp. 63-83.

[3] Fraser, D.M., Pillay, R., Tjatindi, L., Case, J.M. "Enhancing the learning of fluid mechanics using computer simulations.” Journal of Engineering Education. Volume 96, No. 4, 2007, pp. 381-388.

[4] Tannehill, J.C., Anderson, D.A., Pletcher, R.H. Computational Fluid Mechanics and Heat Transfer. $2^{\text {nd }}$ Ed. Taylor \& Francis. 1997. Philadelphia, PA.

[5] Malalasekera, W., Verseeg, H.K. An Introduction to Computational Fluid Dynamics: The finite volume method. Longman Scientific \& Technical. 1995. New York, NY.

[6] Medina, R., Okcay, M., Menezes, G., Pacheco-Vega, A. "Implementation of Particle Image Velocimetry in the Fluid Mechanics Laboratory." In: Proceedings of the 2011 PSW American Society for Engineering Education Conference, Fresno, CA, USA, 2011, pp. 42-50.

[7] Pnueli, D., Gutfinger, C. Fluid Mechanics. Cambridge University Press. 1992. Cambridge, UK.

[8] Ferziger, J. H., Peric, M. Computational Methods for Fluid Dynamics, 3rd Ed. 2001. Springer, New York, NY.

[9] Jasak, H. "Error Analysis and Estimation for the Finite Volume Method with Applications to Fluid Flows", Ph.D. Thesis, Imperial College, London, 1996

[10] Patankar, S.V. "A calculation procedure for two-dimensional elliptic situations." Numerical Heat Transfer, Volume 4, 1981, pp. 409-425.

[11] Galpin, P.F., Van Doormaal, J.P., Raithby, G.D. "Solution of the incompressible mass and momentum equation by application of a coupled equation line solver." International Journal for Numerical Methods in Fluids, Volume 5, 1985, pp. 615-625.

[12] Galpin, P.F., Raithby, G.D. "Treatment of non-linearities in the numerical solution of the incompressible Navier-Stokes equations.” International Journal for Numerical Methods in Fluids, Volume 6, 1986, pp. 409-426

[13] Rusche, H. "Computational fluid dynamics of dispersed two-phase Flows at High Phase Fractions," Ph.D. Thesis, Imperial College, London, 2002

[14] Zhao, Y., Zhang, B. "A high-order characteristics upwind FV method for incompressible flow and heat transfer simulation on unstructured grids." Computer Methods in Applied Mechanics and Engineering, Volume 190, 2000, pp. 733-756. 